\title{
Biliary Mucinous Cystadenoma: A Review of the Literature
}

\author{
Leon D. Averbukh* ${ }^{1}$, David C. Wu ${ }^{1}$, Woo Cheal $\mathrm{Cho}^{2}$ and George Y. $\mathrm{Wu}^{1}$ \\ ${ }^{1}$ Department of Medicine, Division of Gastroenterology-Hepatology, University of Connecticut Health Center, Farmington, CT, \\ USA; ${ }^{2}$ Department of Pathology, Hartford Hospital, Hartford, CT, USA
}

\begin{abstract}
Biliary mucinous cystadenomas are cystic neoplasms commonly mistaken for simple cysts. They are rare and generally benign tumors, often incidentally found on imaging and during unrelated surgical interventions. They tend to be slow growing though may reach symptomatic dimensions. Misdiagnosis of biliary mucinous cystadenomas may have serious consequences secondary to their potential for malignant transformation into biliary mucinous cystadenocarcinomas. Here, we review the epidemiology, etiology, pathology, diagnostic modalities, histology, and available treatment methods for mucinous cystadenomas reported in current literature.

Citation of this article: Averbukh LD, Wu DC, Cho WC, Wu GY. Biliary mucinous cystadenoma: A review of the literature. J Clin Transl Hepatol 2019;7(2):149-153. doi: 10.14218/JCTH.2019.00017.
\end{abstract}

\section{Introduction}

Biliary cystadenomas are a family of rare cystic neoplasms of the biliary ductal system. Comprised of two subvariantsserous (more common) and mucinous (less common)-these tumors are considered benign but premalignant. Biliary mucinous cystadenomas (BMCA) account for less than $5 \%$ of all hepatic cystic neoplasms. ${ }^{1}$ Due to their malignant transformation potential, BMCAs should undergo evaluation for surgical intervention whether symptomatic or not. ${ }^{2}$

In this review, we describe the epidemiology and distinguishing features of hepatic BMCAs on histology and imaging. We further discuss the controversy surrounding the lesion's proposed origins and identify treatment options and their respective efficacies.

\section{Epidemiology}

The incidence of biliary cystadenomas is 1 in 20,000-100,000, while the risk of malignant transformation to adenocarcinoma has been reported to be as high as $20-23 \% .{ }^{3}$ BMCAs are most commonly (85-95\% of reported cases) seen in Caucasian females in middle age (55-65 years old). ${ }^{4}$ The tumors are

Keywords: Biliary mucinous cystadenoma; Biliary mucinous cystadenocarcinoma; Cystic neoplasm.

Abbreviations: BMCA, biliary mucinous cystadenoma; BMCAC, biliary mucinous cystadenocarcinoma; CA, cancer antigen; CT, computed tomography; MRI, magnetic resonance imaging; OS, ovarian stroma; US, ultrasound.

Received: 13 April 2019; Revised: 25 May 2019; Accepted: 11 June 2019

*Correspondence to: Leon D. Averbukh, Department of Medicine, Division of Gastroenterology-Hepatology, University of Connecticut Health Center, 236 Farmington Ave., Farmington, CT 06030, USA. E-mail: Averbukh@uchc.edu subcategorized into two subgroups: those that have mesenchymal tissue resembling ovarian stroma (OS) on histology and those that do not (nonOS). While the former variant is much more common and seen exclusively in women, the latter has an equal distribution between men and women and is associated with an overall poorer prognosis and higher rate of recurrence. ${ }^{4,5}$ One study showed that women with OS variant BMCA were on average younger in age than their nonOS counterparts at the time of presentation/diagnosis and were generally within child-bearing age. ${ }^{6}$

The incidence of malignant biliary mucinous cystadenocarcinoma (BMCAC) is equal between males and females and the average age of patients at presentation is $10-20$ years older than for those with BMCA. ${ }^{6-8}$ Though BMCA tumors have great variability in size at the time of presentation, they are frequently between 2-25 cm in diameter. ${ }^{9}$ Most BMCAs arise from the intrahepatic biliary ducts and they are commonly found in the posterior segment of the right liver lobe, likely due to the fact that the right lobe contains the most hepatic tissue. ${ }^{9}$ While BMCA is a type of proliferative epithelial tumor, its progression is generally slow, though this varies from patient to patient. One study reported significant abdominal girth increase in patients with BMCA in as little as 5 weeks to as long as over 12 years. ${ }^{5}$ Additionally, tumor growth rates may be inconsistent longitudinally, as patients have been reported to have slow tumor growth punctuated by periods of rapid growth due to sudden cystic fluid accumulation. ${ }^{7}$

\section{Etiology}

While the origin of BMCA is still debated, findings in experimental studies support the involvement of an acquired process through a superficial injury and an inductive reactive process. ${ }^{5,10}$ Alternative theories of inductive disease processes include congenital variants such as the presence of aberrant bile duct nests as well as von Meyenberg complexes (bile duct hamartomas and biliary microhamartomas in the liver composed of ectopic embryonic tissues). ${ }^{11}$ The presence of endocrine cells in about $50 \%$ of BMCAs suggests an origin from intrahepatic peribiliary glands. ${ }^{3}$ However, the theory of congenital disease origin is not limited to the primordial gallbladder, as other studies have suggested that the ectopic tissue may originate from the ovaries and embryonic foregut. ${ }^{12,13}$

The ovarian origin of disease has been supported by immunohistochemical studies of BMCAs, which have shown mesenchymal stromal cells expressing estrogen and progesterone receptors. This is supported by the finding that women on oral estrogen contraceptive therapy have experienced rapidly growing lesions, suggesting that the estrogen therapy acts as a hormonal tumor promoter. ${ }^{3,14}$ Further evidence supporting a gonadal origin for the lesions include the 
finding that phenotypically similar mucinous cystadenomas are found in areas closest to the embryonic gonads, such as the liver, pancreas, and spleen. ${ }^{2}$ Gonadal fusion to the liver and spleen has also been well documented during pediatric surgeries. $^{2}$

\section{Pathology/symptomatology}

While BMCAs are often asymptomatic and diagnosed as incidental findings on imaging/surgical exploration, those patients that are symptomatic at the time of presentation generally complain of vague abdominal symptoms. ${ }^{8}$ Symptoms associated with BMCAs include palpable abdominal mass (the most commonly reported symptom, being in roughly $60 \%$ of cases) as well as abdominal discomfort that is either diffuse or limited to the right upper quadrant or the epigastric area. ${ }^{15}$ Less commonly, in cases involving compression of the stomach and duodenum, patients report nausea/ vomiting, dyspepsia, anorexia, and weight loss. ${ }^{15}$ Biliary obstruction secondary to either tumor compression of the biliary tract or tumor-produced mucin plugging the biliary tract may lead to symptoms of jaundice, biliary colic, nausea, fever, chills, itching, and steatorrhea. ${ }^{1}$ Obstructive jaundice on presentation is significantly more common in cases of extrahepatic BMCA rather than of intrahepatic lesions. ${ }^{1}$ Additionally, in cases where inferior vena caval or hepatic vein compression occurs, patients may present with abdominal swelling and ascites. ${ }^{7} 35 \%$ of patients with BMCA experience complications secondary to obstruction such as cholangitis, rupture, and intracystic hemorrhage, or due to malignant transformation. ${ }^{16}$

\section{Diagnostic imaging methods and findings}

The choice of imaging in patients with suspected hepatic cysts is dependent on patient risk, screening imaging availability, cost effectiveness, safety, and diagnostic accuracy. In most cases of BMCA, the lesion is identified incidentally by ultrasound (US), noncontrast computed tomography (CT) of the abdomen, or during unrelated surgical procedures. ${ }^{17}$ For those patients with suspected BMCA and no prior imaging, the initial screening should be US, as its sensitivity is approximately $90 \% .{ }^{18}$ Findings on US include an anechoic lesion with thickened and irregular walls and internal septations (Fig. 1). Unlike US, endoscopic US with fine needle aspiration is not used regularly in diagnosis of BMCA, and at present no literature reporting on its diagnostic efficacy is available.

In medically stable patients who have undergone prior noncontrast CT or US, CT with contrast is recommended for differentiation of BMCA from solitary bile duct cysts. ${ }^{17}$ One study found that by using a 2-of-5 characteristic selection criteria (presence of septa, central septa, mural nodules, bile duct dilation either upstream or downstream), they were able to successfully differentiate between mucinous cystic neoplasms and solitary bile duct cysts with a sensitivity of $87 \%$ and a specificity of $87 \% .{ }^{17}$ While CT is not as sensitive for detecting septations in cystic lesions when compared to sonography, it is superior in demonstrating accurate size and anatomic extent of the lesion. ${ }^{5}$ Additionally, BMCAs are usually isodense to water (less than 30 Hounsfield units) and display nodular areas of enhancement on CT with contrast ${ }^{19}$ (Table 1).

Magnetic resonance imaging (MRI) has the added benefit of demonstrating anatomic relationships within the liver to a higher degree than on CT, a quality which can aid in surgical planning. ${ }^{20}$ Findings on MRI depend on the type of weighted images, with T1

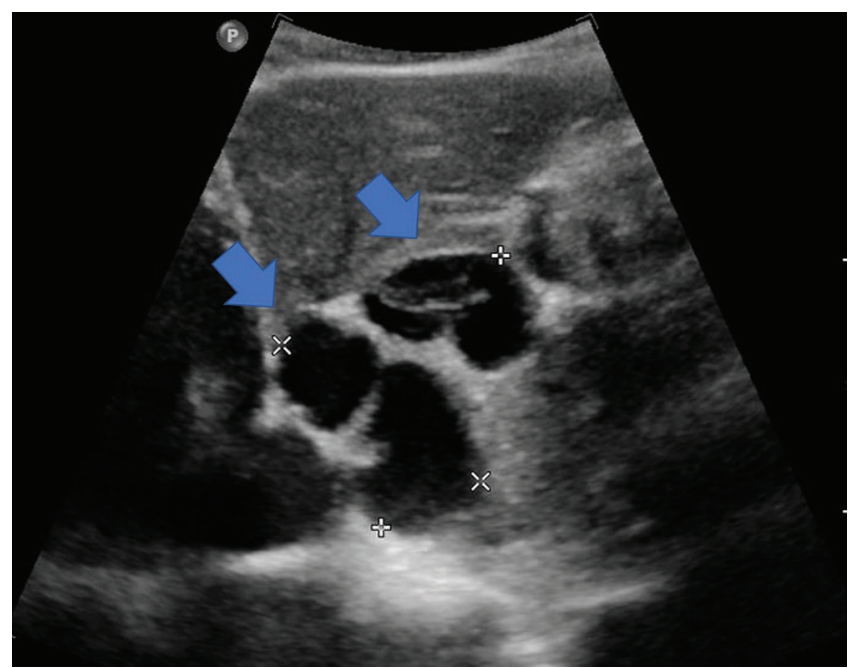

Fig. 1. BMCA with typical ultrasound findings of an anechoic lesion with thickened and irregular walls, and internal septations.

showing varying cystic fluid signal intensities and intracystic septations identified on T2-weighted images (Fig. 2).

In cases of intrabiliary lesions or those or those in the proximal biliary tree, magnetic resonance cholangiopancreatography may be helpful in identifying areas of obstruction and cyst communication with the biliary tree. Rarely, endoscopic retrograde cholangiopancreatography may also be employed for lesions communicating with the biliary tree for tissue sampling purposes. ${ }^{8}$

The ability to consistently and accurately differentiate mucinous cystic neoplasms from other solitary cysts using imaging alone has been controversial. One study on a 52patient cohort found that no individual radiological characteristics from US, CT, or MRI successfully differentiated between the two lesion types. ${ }^{21}$

\section{Differential diagnosis}

Hepatic cystic neoplasms can be simple or complex, with complex cysts characterized by septations, debris-containing

Table 1. Appearance of biliary mucinous cystadenoma on various imaging modalities

\begin{tabular}{|c|c|}
\hline Imaging modality & $\begin{array}{l}\text { Appearance of biliary mucinous } \\
\text { cystadenoma }\end{array}$ \\
\hline Ultrasound & $\begin{array}{l}\text { Anechoic } \\
\text { Thickened irregular walls and } \\
\text { multiple internal septations } \\
\text { Papillary in-folding, mural nodules }\end{array}$ \\
\hline $\begin{array}{l}\text { Computed } \\
\text { tomography }\end{array}$ & $\begin{array}{l}\text { Multicystic and multiseptated lesion } \\
\text { With contrast, displays isodensity to } \\
\text { water (less than } 30 \text { Hounsfield units) } \\
\text { Displays nodular areas of } \\
\text { enhancement }\end{array}$ \\
\hline $\begin{array}{l}\text { Magnetic } \\
\text { resonance } \\
\text { imaging }\end{array}$ & $\begin{array}{l}\text { Multilocular with irregular thick walls } \\
\text { Varying cystic fluid signal intensities } \\
\text { and intracystic septations }\end{array}$ \\
\hline
\end{tabular}




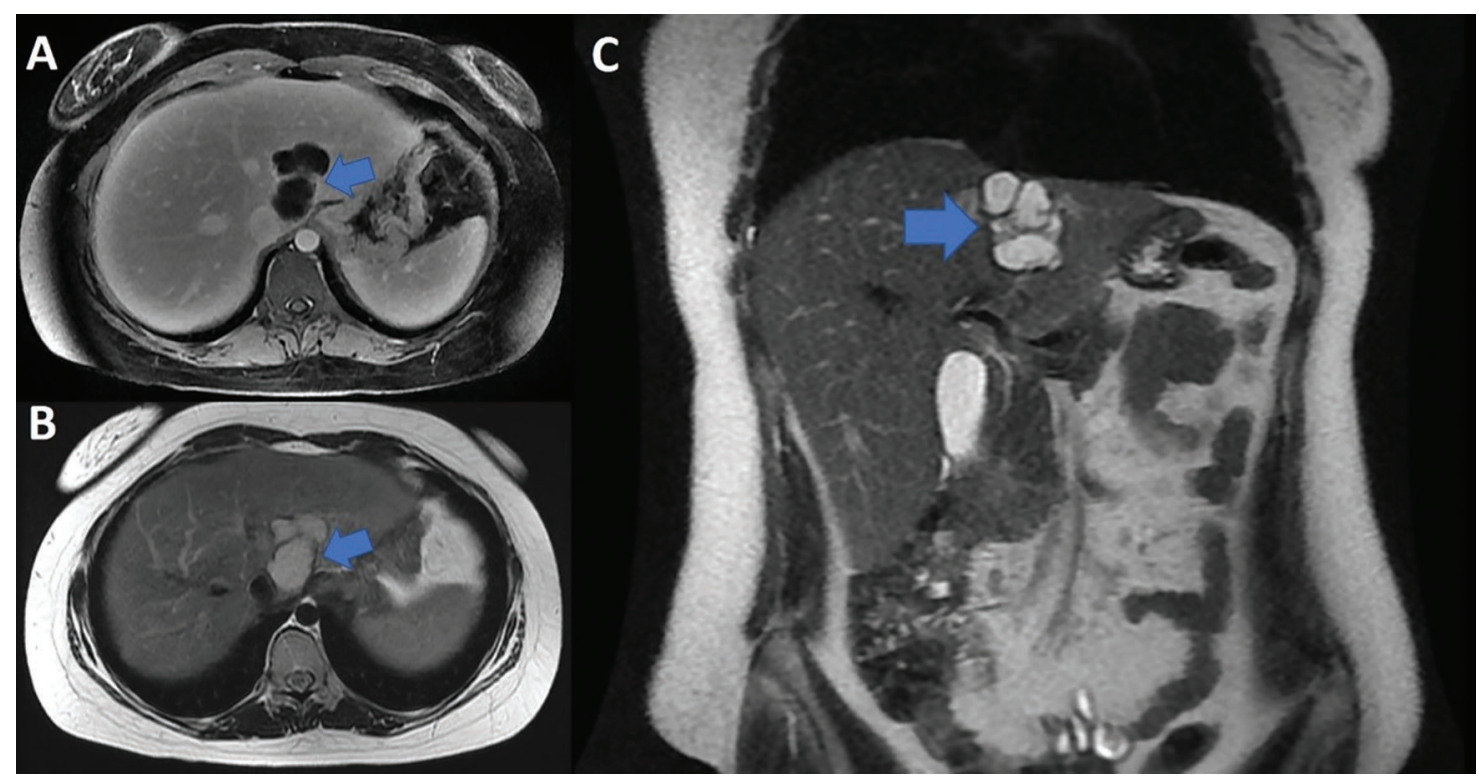

Fig. 2. BMCA findings on magnetic resonance imaging showing a septated and multilobulated lesion on T1-weighted imaging (A) and hyperintense signaling on T2-weighted imaging (B-C).

fluid, hemorrhage, mural thickening, nodularity, or proteinaceous fluid collection. ${ }^{22,23}$ The differential diagnosis for hepatic cystic neoplasms can be categorized by etiology: developmental, inflammatory, neoplastic, traumatic, and miscellaneous ${ }^{22}$ (Table 2). Generally, the presence of septation and septal thickening are significant predictors of possible BMCA. Cysts

Table 2. Differential diagnosis of hepatic cystic neoplasms

\begin{tabular}{|c|c|}
\hline $\begin{array}{l}\text { Hepatic } \\
\text { cystic neoplasms }\end{array}$ & Diagnosis \\
\hline Developmental & $\begin{array}{l}\text { Simple benign cyst } \\
\text { Adult polycystic liver disease } \\
\text { Caroli disease }\end{array}$ \\
\hline \multirow[t]{2}{*}{ Inflammatory } & $\begin{array}{l}\text { Pyogenic } \\
\text { Parasitic } \\
\text { - Entamoeba histolytic } \\
\text { - Hydatid (echinococcal) }\end{array}$ \\
\hline & $\begin{array}{l}\text { Abscess } \\
\text { - Fungal } \\
\text { - Bacterial }\end{array}$ \\
\hline \multirow[t]{2}{*}{ Neoplastic } & $\begin{array}{l}\text { Primary } \\
\text { - Undifferentiated embryonal } \\
\text { carcinoma } \\
\text { - Biliary cystadenoma or } \\
\text { cystadenocarcinoma } \\
\text { - Embryonal sarcoma } \\
\text { - Cavernous hemangioma } \\
\text { - Intraductal papillary neoplasms }\end{array}$ \\
\hline & $\begin{array}{l}\text { Secondary } \\
\text { - Cystic metastasis }\end{array}$ \\
\hline \multirow[t]{2}{*}{ Traumatic } & Hemorrhagic or hematoma \\
\hline & $\begin{array}{l}\text { Seroma } \\
\text { Biloma } \\
\text { Pseudocyst }\end{array}$ \\
\hline
\end{tabular}

that do not have internal septa or papillary projections are more likely to be simple cysts which can be managed conservatively with regularly scheduled follow up. ${ }^{24}$ Other cystic lesions that also have septations, such as liver abscesses and echinococcal cysts, are harder to differentiate from BMCA through imaging alone and may require fine needle aspiration.

On imaging, echinococcal cysts often have debris or daughter cysts within the main cyst, and liver abscesses typically have a more irregular and thicker wall than seen in BMCA. ${ }^{5}$ Hemorrhagic cysts may also appear nodular with septations on US and weighted MRI, due to intracystic blood clots. However, unlike with BMCA, hemorrhagic cysts often appear to be homogenous on CT as the latter have decreased sensitivity for intracystic contents. ${ }^{25}$

Other multiloculated cystic lesions include mesenchymal hamartomas and embryonal sarcomas. While they have a similar appearance to BMCAs on imaging, demographic characteristics of patients presenting with these lesions (children and young adults rather than middle-aged women) differ. For those patients with BMCA aspiration of cystic fluid generally yields bile-tinged mucin helping to differentiate the lesion from other cystic lesions, such as hemorrhagic and parasitic. Intraductal papillary mucosal neoplasms may be the hardest lesions to distinguish from BMCAs, as they are also mucin-producing complex cysts and ultimately require histological differentiation postbiopsy. ${ }^{2}$

\section{Histopathology}

BMCAs are generally large and multiloculated, with internal septations surrounded by cellular fibrostroma. Most commonly, they contain clear or bile-tinged mucinous fluid, though some have been reported to be purulent and proteinaceous as well. ${ }^{13}$ The presence of blood within the cyst is a concerning finding and may signify a malignant component. ${ }^{25}$ The septations may also present with calcifications, though this finding is frequently a sign of benignity. BMCAs are true proliferative epithelial tumors and consist of a layer of biliary 
type cuboidal-to-tall columnar, mucin-producing, nonciliated epithelial cells, with papillary necrosis. ${ }^{23,26,27}$ In patients with the OS subvariant, there is an additional mesenchymal stroma layer between the inner epithelial lining and an outer connective tissue capsule (Fig. 3). In those patients with the nonOS variant, a hyaline stroma layer exists in place of the mesenchymal stroma. ${ }^{4}$

BMCAs have been reported to show reactivity to markers common for epithelial lesions arising from the abdominal cavity, namely cytokeratins (CAM5.2, AE1, AE3), cancer antigen (CA) 19-9, epithelial membrane antigen, and carcinoembryonic antigen. ${ }^{26}$ However, these findings have been inconsistent and do not allow for definitive differentiation between BMCA and BMCAC. ${ }^{21}$ Cystic fluid aspirate CA 19-9 may be helpful in differentiating BMCA from other cystic lesions, however, as levels of CA 19-9 in BMCA are on average significantly higher than in other lesions. ${ }^{28}$ While monitoring trends of serum CA $19-9$ at regularly spaced intervals has been suggested for postexcisional tumor resolution, this strategy is not recommended for long-term monitoring because immunoreactivity to the marker has been noted to be lost upon malignant transformation to BMCAC. $^{29}$

BMCACs are difficult to distinguish from BMCAs due to a lack of consistent and significant differences in immunohistochemical markers. On histology, however, BMCAC demonstrates characteristics of cytologically malignant epithelium, consisting of multiple layers with nuclear pleomorphism, loss of cellular polarity, and papilla of fibrous tissue invading through the basement membrane. ${ }^{23,26,30}$
Averbukh L.D. et al: Biliary mucinous cystadenoma: A review

\section{Treatment and prognosis}

For those who have suspected or confirmed diagnosis of BMCA, active intervention rather than tumor monitoring is recommended, due to the potential for malignant transformation. Treatment of the condition ranges from simple aspiration to liver transplantation. ${ }^{8}$ More conservative therapies, such as cyst aspiration, laparoscopic fenestration, internal drainage by roux-en-Y, narrow margin cyst excision, sclerotherapy, and cyst decompression have been reported. However, concerns for high rate of tumor recurrence (as high as $80 \%$ for partial cyst excision and $100 \%$ for cyst aspiration) as well as complications (including biliary obstruction, secondary infection or sepsis, rupture, and hemorrhage) have prevented their regular use and are thus not recommended. ${ }^{4,7}$ Wide-margin $(2 \mathrm{~cm})$ resection is considered the treatment of choice, although rates of recurrence have been reported to be between $5-20 \% .^{7}$ It is unclear why such variability in neoplasm recurrence rates exists for wide-margin cyst excisions, though it may be in part secondary to initial tumor resection completeness. In cases where wide-margin resection is not possible, such as in centrally-located lesions involving central vascular or biliary structures, enucleation may be performed instead. ${ }^{31}$

In cases such as giant single and double lobar BMCAs, where the size or quantity of the cysts makes excision impossible, as well as in cases where prior surgical intervention was unsuccessful or injurious, lobectomy and even liver transplantation may be considered. ${ }^{31}$ Aside from the need for a liver donor, such radical therapy requires patients to undergo life-long immunosuppressive therapy. ${ }^{7}$ Therefore,
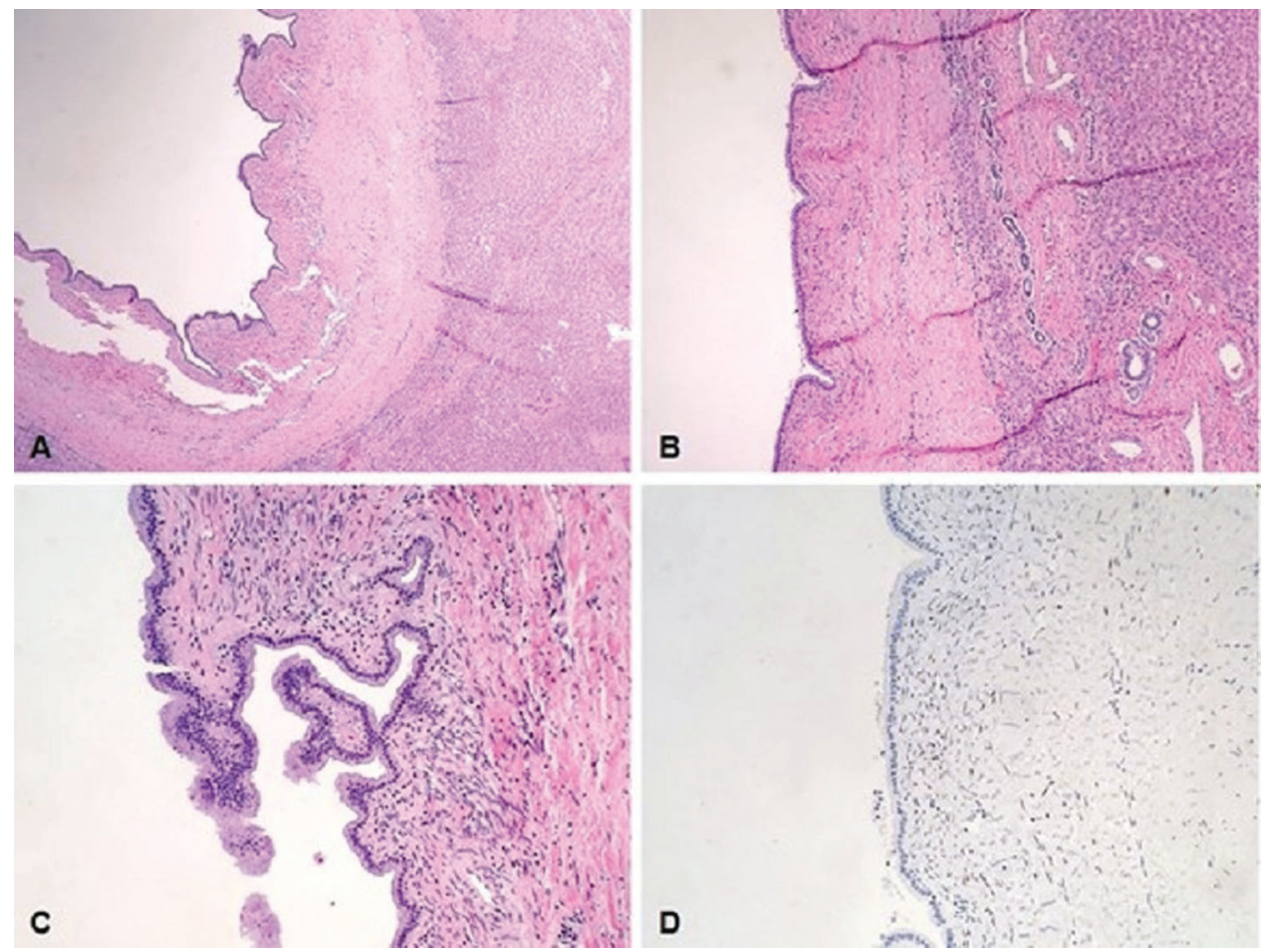

Fig. 3. Histologic features of biliary cystadenoma. A, B: Histologically, biliary cystadenoma is characterized by multiloculated cysts lined by a monolayer of cuboidal, columnar or attenuated mucinous epithelium and often surrounded by a thick hyalinized fibrous cystic wall. C, D: Occasionally, the subepithelial stroma displays a proliferation of spindle cells, reminiscent of ovarian-type stroma, which usually expresses estrogen receptors, progesterone receptors (not shown), and inhibin (not shown). Hematoxylin and eosin staining for all. Original magnification of $\times 40, \times 100, \times 200$, and $\times 200$ for $A-D$, respectively. 
the risk and benefits of intervention should be weighed and discussed with the patient and a multidisciplinary review of the case must be conducted. ${ }^{6}$

\section{Conclusions}

BMCAs are rare, generally slow-growing, benign lesions that are often asymptomatic and incidentally found. While most commonly identified in middle-aged Caucasian females, depending on the BMCA subvariant, they can also be found in males. The propensity for BMCAs to grow to symptomatic sizes as well as their ability to undergo malignant transformation are of concern. Although making the diagnosis by imaging alone is difficult, an approach using multiple imaging modalities, such as US, CT and MRI, aids in the identification of septations and other key cystic features. Cystic fluid aspiration may also help in differentiating BMCAs from other etiologies, as they often contain bile-tinged mucin. Ultimately, biopsy and histological analysis remains the gold standard for BMCA diagnosis and differentiation from BMCAC. Histologically, BMCAs typically display biliary glandular tissue with or without a subepithelial layer of densely organized spindle-shaped cells known as "ovarian-like-stroma".

Those with the nonOS variant are at higher risk for tumor recurrence and have an overall poorer prognosis. Due to the high propensity for recurrence postintervention and its malignant transformation in roughly 1 in 5 diagnosed cases of BMCAs, it is recommended that patients with diagnosed BMCA isolated to a single hepatic lobe and not located within or communicating with the biliary tree, undergo complete cyst excision with wide margins. For those with difficult lesions, including "giant" cysts or centrally-located lesions involving the biliary tree and its vasculature, alternative therapies including enucleation and liver transplant should be considered.

\section{Conflict of interest}

The authors have no conflict of interests related to this publication.

\section{Author contributions}

Wrote the manuscript (LDA, DCW), interpreted the pathology results (WCC), developed the idea for the article and critically revised it (GYW).

\section{References}

[1] Tsepelaki A, Kirkilesis I, Katsiva V, Triantafillidis JK, Vagianos C. Biliary Cystadenoma of the liver: Case report and systematic review of the literature. Ann Gastroenterol 2009;22:278-283.

[2] Erdogan D, Kloek J, Lamers WH, Offerhaus GJ, Busch OR, Gouma DJ, et al. Mucinous cystadenomas in liver: management and origin. Dig Surg 2010;27: 19-23. doi: 10.1159/000268110.

[3] Koffron A, Rao S, Ferrario M, Abecassis M. Intrahepatic biliary cystadenoma: role of cyst fluid analysis and surgical management in the laparoscopic era. Surgery 2004;136:926-936. doi: 10.1016/j.surg.2004.06.031.

[4] Manouras A, Lagoudianakis E, Alevizos L, Markogiannakis H, Kafiri G, Bramis $\mathrm{C}$, et al. Laparoscopic fenestration of multiple giant biliary mucinous cystadenomas of the liver. World J Gastroenterol 2008;14:4257-4259. doi: 10 . 3748/wjg.14.4257.

[5] Ishak KG, Willis GW, Cummins SD, Bullock AA. Biliary cystadenoma and cystadenocarcinoma: report of 14 cases and review of the literature. Cancer 1977;39:322-338. doi: 10.1002/1097-0142(197701)39:1<322: AID-CNCR2820390149>3.0.CO;2-P.

[6] Wheeler DA, Edmondson HA. Cystadenoma with mesenchymal stroma (CMS) in the liver and bile ducts. A clinicopathologic study of 17 cases,
4 with malignant change. Cancer 1985;56:1434-1445, doi: 10.1002/10970142(19850915)56:6<1434::AID-CNCR2820560635>3.0.CO;2-F

[7] Grubor NM, Colovic RB, Atkinson HD, Micev MT. Giant biliary mucinous cystadenoma of the liver. Ann Hepatol 2013;12:979-983. doi: 10.1016/s16652681(19)31306-7.

[8] Soares KC, Arnaoutakis DJ, Kamel I, Anders R, Adams RB, Bauer TW, et al. Cystic neoplasms of the liver: biliary cystadenoma and cystadenocarcinoma. J Am Coll Surg 2014;218:119-128. doi: 10.1016/j.jamcollsurg.2013.08.014.

[9] Lind DS, Adolph V, Parker GA. Mucinous biliary cystadenoma: a case report and review of the literature. J Surg Oncol 1992;51:207-210. doi: 10 . $1002 /$ jso.2930510316.

[10] Cruickshank AH, Sparshott SM. Malignancy in natural and experimental hepatic cysts: experiments with aflatoxin in rats and the malignant transformation of cysts in human livers. J Pathol 1971;104:185-190. doi: 10. 1002/path. 1711040305 .

[11] Walt AJ. Cysts and benign tumors of the liver. Surg Clin North Am 1977;57: 449-464. doi: 10.1016/S0039-6109(16)41194-1.

[12] Kazama S, Hiramatsu T, Kuriyama S, Kuriki K, Kobayashi R, Takabayashi N, et al. Giant intrahepatic biliary cystadenoma in a male: a case report, immunohistopathological analysis, and review of the literature. Dig Dis Sci 2005; 50:1384-1389. doi: 10.1007/s10620-005-2791-6.

[13] Florman SS, Slakey DP. Giant biliary cystadenoma: case report and literature review. Am Surg 2001;67:727-732.

[14] Suyama Y, Horie Y, Suou T, Hirayama C, Ishiguro M, Nishimura O, et al. Oral contraceptives and intrahepatic biliary cystadenoma having an increased level of estrogen receptor. Hepatogastroenterology 1988;35:171-174.

[15] Short WF, Nedwich A, Levy HA, Howard JM. Biliary cystadenoma. Report of a case and review of the literature. Arch Surg 1971;102:78-80. doi: 10 . 1001/archsurg.1971.01350010080021.

[16] Rutledge JN, Pratt MC, Taupmann RE. Biliary cystadenoma mistaken for an echinococcal cyst. South Med J 1983;76:1575-1577. doi: 10. 1097/00007611-198312000-00032

[17] Kim HJ, Yu ES, Byun JH, Hong SM, Kim KW, Lee JS, et al. CT differentiation of mucin-producing cystic neoplasms of the liver from solitary bile duct cysts. AJR Am J Roentgenol 2014;202:83-91. doi: 10.2214/AJR.12.9170.

[18] Lantinga MA, Gevers TJ, Drenth JP. Evaluation of hepatic cystic lesions. World J Gastroenterol 2013;19:3543-3554. doi: 10.3748/wjg.v19.i23.3543.

[19] Mortele KJ, Ros PR. Benign liver neoplasms. Clin Liver Dis 2002;6:119-145. doi: 10.1016/S1089-3261(03)00069-2.

[20] Delis SG, Touloumis Z, Bakoyiannis A, Tassopoulos N, Paraskeva K, Athanassiou $\mathrm{K}$, et al. Intrahepatic biliary cystadenoma: a need for radical resection. Eur J Gastroenterol Hepatol 2008;20:10-14. doi: 10. 1097/MEG.0b013e3282f16a76.

[21] Labib PL, Aroori S, Bowles M, Stell D, Briggs C. Differentiating simple hepatic cysts from mucinous cystic neoplasms: Radiological features, cyst fluid tumour marker analysis and multidisciplinary team outcomes. Dig Surg 2017;34:36-42. doi: 10.1159/000447308.

[22] Vachha B, Sun MR, Siewert B, Eisenberg RL. Cystic lesions of the liver. AJR Am J Roentgenol 2011;196:W355-W366. doi: 10.2214/AJR.10.5292.

[23] Zen Y, Pedica F, Patcha VR, Capelli P, Zamboni G, Casaril A, et al. Mucinous cystic neoplasms of the liver: a clinicopathological study and comparison with intraductal papillary neoplasms of the bile duct. Mod Pathol 2011;24: 1079-1089. doi: 10.1038/modpathol.2011.71.

[24] Choi BI, Lim JH, Han MC, Lee DH, Kim SH, Kim YI, et al. Biliary cystadenoma and cystadenocarcinoma: CT and sonographic findings. Radiology 1989; 171:57-61. doi: 10.1148/radiology.171.1.2648477.

[25] Del Poggio P, Buonocore M. Cystic tumors of the liver: a practical approach. World J Gastroenterol 2008;14:3616-3620. doi: 10.3748/wjg.14.3616.

[26] Devaney K, Goodman ZD, Ishak KG. Hepatobiliary cystadenoma and cystadenocarcinoma. A light microscopic and immunohistochemical study of 70 patients. Am J Surg Pathol 1994;18:1078-1091. doi: 10.1097/00000478199411000-00002.

[27] Ferrell LD. Benign and malignant tumors of the liver. In: Odze R, Goldblum J, editors. Surgical pathology of the GI tract, liver, biliary tract and pancreas. Philadelphia: Saunders; 2004. p. 1015-1016.

[28] Lee JH, Chen DR, Pang SC, Lai YS. Mucinous biliary cystadenoma with mesenchymal stroma: expressions of CA $19-9$ and carcinoembryonic antigen in serum and cystic fluid. J Gastroenterol 1996;31:732-736. doi: 10. $1007 /$ bf02347626.

[29] Sirén J, Kärkkäinen P, Luukkonen P, Kiviluoto T, Kivilaakso E. A case report of biliary cystadenoma and cystadenocarcinoma. Hepatogastroenterology 1998;45:83-89.

[30] Terada T, Nakanuma Y, Ohta T, Nagakawa T, Motoo $Y$, Harada A, et al. Mucinhistochemical and immunohistochemical profiles of epithelial cells of several types of hepatic cysts. Virchows Arch A Pathol Anat Histopathol 1991;419: 499-504. doi: 10.1007/bf01650679.

[31] Romagnoli R, Patrono D, Paraluppi G, David E, Tandoi F, Strignano P, et al. Liver transplantation for symptomatic centrohepatic biliary cystadenoma. Clin Res Hepatol Gastroenterol 2011;35:408-413. doi: 10.1016/j.clinre. 2011.03.014. 